\title{
Molecular stratification and repair defects: revealing hidden treasures
}

Most men with metastatic castrationresistant prostate cancer ( $\mathrm{mCRPC}$ ) succumb to their disease, which is highly heterogeneous. Molecular aberrations in men with advancedstage disease have been associated with sensitivity to platinum-based chemotherapy and poly(ADP-ribose) polymerase-1 (PARP) inhibitors. The PARP protein is involved in multiple aspects of DNA repair, and the PARP inhibitor olaparib has been approved for the treatment of advanced-stage ovarian cancer. As some prostate cancers have DNA-repair defects, the potential for exploiting a synthetic lethality approach was explored in an investigator-led phase II trial recently published in the New England Journal of Medicine. PARP inhibitors have shown efficacy in men with mCRPC and germline BRCA2 mutations; thus, the possibility of efficacy in sporadic cases was analysed.

Johann de Bono, senior author of the study, explains the background that led to the trial. "We hypothesized that some prostate cancers had DNA-repair defects. This was based on the presence of platinum sensitivity in some cancers, PARP sensitivity, and that BRCA2-

germline-mutated advanced-stage prostate cancers are sensitive to PARP inhibitors". In the TOPARP-A (Trial of PARP Inhibition in Prostate Cancer), open-label trial, men with mCRPC were treated with olaparib, and fresh tumour biopsy samples were taken for biomarker assessment of both germline and somatic DNA mutations. Each enrolled patient received $400 \mathrm{mg}$ olaparib twice daily until evidence of radiological progression, clinical progression, or unacceptable toxicity. The primary end point of the trial was response rate, according to the following criteria: RECIST criteria version 1.1 , a decline in serum PSA levels of $\geq 50 \%$, or a confirmed reduction in the circulating tumour cell (CTC) count from $\geq 5$ cells per $7.5 \mathrm{ml}$ of blood to $<5$ cells per $7.5 \mathrm{ml}$.

A total of 50 patients were enrolled from seven centres. All patients had received previous treatment with docetaxel, $98 \%$ had received abiraterone or enzalutamide, and 58\% had received cabazitaxel. All patients on the trial received at least one dose of olaparib. Paired biopsy samples from before and after treatment with olaparib were available for biomarker assessment; 28 patients had a bone-marrow biopsy and 22 received image-guided biopsy. Nextgeneration sequencing assays revealed homologous deletions, mutations, or both in DNA-repair genes, which included BRCA1 and BRCA2, ATM, Fanconi anaemia genes, and CHEK2. Of the patients with aberrations in these genes (16 out of 49 ), $88 \%$ (14) had a response to olaparib, including all patients with $B R C A 2$ loss. Overall, 11 out of 49 patients (22\%) had a response according to the PSA criteria.

Importantly, the study showed that the 16 men with aberrations in DNA-repair genes had a significantly higher response rate $(P<0.001)$ : only 2 out of 33 men classified as biomarker-negative had a response. Moreover, radiological-progressionfree survival was significantly longer in the biomarker-positive group than in the biomarker-negative group (9.8 months versus 2.7 months; $P<0.001)$. Overall survival was also longer in the biomarker-positive group compared with the biomarkernegative group (13.8 months versus 7.5 months; $P=0.05$ ). All patients with BRCA2 loss had serum PSA levels that declined by at least $50 \%$ from baseline levels. The sensitivity of detection of the DNA-repair alterations was $88 \%$, and the specificity was $94 \%$. The most-common grade 3 or 4 adverse effects were anaemia, fatigue, neutropenia and leukopenia; dose reduction to $300 \mathrm{mg}$ twice daily was required in 13 patients.

The most crucial finding from this study is that men with a common subset of prostate cancer can, for the first time, be molecularly stratified for treatment. This subset - characterized by defects in DNA repair - comprises $25-30 \%$ of all men with sporadic mCRPC.

The results from this study indicate that this approach of molecular stratification and next-generation sequencing analysis of biopsy samples is feasible. Furthermore, the antitumour activity of olaparib correlated with concomitant declines in serum PSA levels, CTC counts and radiological response. Platinum-based chemotherapy is usually not given for mCRPC as phase II trial data did not demonstrate a survival benefit in unselected patients. In light of the mechanisms of action of platinumbased agents, there is a strong rationale for platinum sensitivity in patients with DNA-repair defects; thus, platinum-based chemotherapy could be combined with olaparib. Dr de Bono summarizes future clinical and research goals: "we are now conducting validation studies to confirm the biomarker suite, and registration phase III studies are also being planned."

Lisa Hutchinson

This article was first published in

Nature Reviews Clinical Oncology doi:10.1038 nrclinonc.2015.205

ORIGINAL ARTICLE Mateo, J. M. et al. DNArepair defects and olaparib in metastatic prostate cancer. N. Engl. J. Med. 373, 1697-1708 (2015) 